\title{
Large-Eddy Simulation of a Turbulent Lifted Flame in a Vitiated Co-Flow
}

\author{
Matthias Ihme* and Yee Chee See ${ }^{\dagger}$ \\ University of Michigan, Ann Arbor, MI 48109-2140
}

\begin{abstract}
Large-eddy simulation (LES) of a lifted flame in a vitiated co-flow has been performed using an unsteady flamelet/progress variable (UFPV) model. This model is an extension to the steady flamelet/progress variable approach, and describes the transient autoignition process of the lifted flame through the unsteady flamelet model. The particular advantage of this model is that it eliminates the flamelet time scale, and all thermochemical quantities are parameterized by mixture fraction, reaction progress parameter, and stoichiometric scalar dissipation rate. For application to LES, a presumed probability density function closure is employed, in which a beta-distribution is used for the mixture fraction, a statistically most-likely distribution is employed for the reaction progress parameter, and the distribution of the stoichiometric scalar dissipation rate is modeled by a Dirac delta function. Compared to the steady flamelet/progress variable model, predictions from the UFPV model show significant improvements, and the spatial evolution of the flame ignition process and lift-off height is in good agreement with experimental data. Flow field structure, statistical results, and scatter data are compared with experimental data, and potential improvements of the model are discussed.
\end{abstract}

\section{Introduction}

$\mathrm{T}$ HE development of advanced combustion systems is mainly controlled by the objective to increase fuel efficiency and to reduce emissions of pollutants such as carbon monoxide, unburned hydrocarbons, and nitrogen oxides $\left(\mathrm{NO}_{x}\right)$. To address these issues, novel combustion strategies have been developed, involving the combustion of lean and diluted fuel-air mixtures. Dilution of the fuel-air mixture in internal combustion engines and furnaces is frequently accomplished by recirculating burned gases, which leads to significant reductions in nitric oxide (NO) emissions due to the decrease in peak combustion temperature. In addition to the pollutant reduction, the dilution with hot combustion products can also lead to improved flame stability. ${ }^{1}$

Despite its enormous potential, combustion of lean and diluted mixtures introduced additional challenges. In particular, the dilution of reactants with inert combustion products can lead to a reduction in the characteristic Damköhler number, so that - unlike to conventional diffusion flames, in which the combustion process is primarily mixing-controlled - the reaction kinetics becomes increasingly important. As such, the stability and characteristics of the flame becomes particularly sensitive to variations in fuel composition and operating conditions. Therefore, ignition mechanisms in such flames play a critical role and are directly affected by the turbulence/chemistry interaction.

Autoignition of a fuel mixture in a hot environment is typically initiated in localized regions of low scalar dissipation rate having a mixture composition that favors short ignition times. Since the prediction of autoignition events, however, is strongly dependent on the structure of the surrounding turbulent reacting flow field, combustion models are required that are able to provide an accurate characterization of the spatiotemporal flow field. Although large-eddy simulation (LES) techniques have been demonstrated to provide improved predictions for the turbulent mixing process compared to Reynolds-averaged Navier-Stokes (RANS) approaches ${ }^{2}$ these intermittent ignition events typically occur on scales that are computationally not resolved. Therefore, subgrid scale closure models are required to characterize effects of unresolved scales

*Assistant Professor, Department of Aerospace Engineering, University of Michigan, Member AIAA

${ }^{\dagger}$ Research Assistant, Department of Aerospace Engineering, University of Michigan 
and ignition kinetics. The objective of this work is to develop a LES model for the prediction of autoignition in lifted flames. This model is based on the unsteady flamelet formulation ${ }^{3}$ and employs a statistically most-likely probability density function (PDF) as presumed PDF closure model.

The LES autoignition model is applied to a lifted methane/air jet flame, which was experimentally investigated by Cabra et al. ${ }^{4}$ In this configuration, a methane fuel jet is issued into a vitiated co-flow with a temperature of $1,350 \mathrm{~K}$, and the flame is stabilized at a lift-off height of approximately 30 nozzle diameters downstream of the jet exit. Because of its simplified geometry, comprehensive experimental database, and sensitivity of the flame characteristics to operating conditions, including co-flow temperature, and inlet velocities, this flame is of particular interest to the computational combustion community for validation and development of combustion models. Gordon et al. ${ }^{5}$ investigated the transport budget in this lifted methane/air jet flame using a composition PDF approach, and their results indicated that the lifted flame is stabilized by autoignition. Similarly, a joint-scalar transported PDF approach with detailed reaction chemistry coupled with a second moment closure model for the velocity prediction was used by Gkagkas \& Lindstedt ${ }^{6}$ to compute this lifted flame. Their model reproduced the flow field sensitivity to boundary conditions, and the role of hydroperoxyl and formaldehyde species on the ignition kinetics was characterized. Domingo et al. ${ }^{7}$ combined a model for autoignition and partially premixed flame propagation. They applied this model to LES of this vitiated flame, and the reported simulation results were in good agreement with experimental data.

Unlike to these model formulations, in the present work a model for the prediction of autoignition is developed which is based on the unsteady flamelet model for diffusion flames. The mathematical model describing the unsteady flamelet/progress variable formulation and the presumed PDF closure is presented in the next section. The experimental configuration and computational setup are summarized in Sec. III. Computational results obtained from the model are compared with experimental data and the steady flamelet/progress variable model in Sec. IV. The paper finishes with conclusions.

\section{Mathematical Model}

\section{A. Large-eddy Simulation}

In LES, the coherent large scale structures of the turbulent flow are computationally resolved, and effects of the smaller and numerically unresolved scales on the large scales are modeled. The decomposition of the scales is achieved by applying a low-pass filter to the flow field quantities, and in the case of a reacting flow, the Favre-filtered quantity of a scalar $\psi$ is computed as

$$
\widetilde{\psi}(t, \boldsymbol{x})=\frac{1}{\bar{\rho}} \int \rho(t, \boldsymbol{x}) \psi(t, \boldsymbol{x}) G(t, \boldsymbol{x}, \boldsymbol{y} ; \Delta) d \boldsymbol{y},
$$

where $t$ denotes time, $\boldsymbol{x}$ is the spatial coordinate, $\rho$ is the density, and $G$ is the filter kernel, satisfying the normalization condition

$$
\int G(t, \boldsymbol{x}, \boldsymbol{y} ; \Delta) d \boldsymbol{y}=1,
$$

and $\Delta$ denotes the filter size, which corresponds in the case of an implicit LES to the local LES grid size. The residual field is defined as

$$
\psi^{\prime \prime}(t, \boldsymbol{x})=\psi(t, \boldsymbol{x})-\widetilde{\psi}(t, \boldsymbol{x}),
$$

and Favre-filtered quantities are related to Reynolds-filtered quantities by $\bar{\rho} \widetilde{\psi}=\overline{\rho \psi}$.

After applying the filter operator to the instantaneous governing equations describing the conservation of mass and momentum, the Favre-filtered equations can be written as

$$
\begin{aligned}
\widetilde{\mathcal{D}}_{t} \bar{\rho} & =-\bar{\rho} \nabla \cdot \widetilde{\boldsymbol{u}} \\
\bar{\rho} \widetilde{\mathcal{D}}_{t} \widetilde{\boldsymbol{u}} & =-\nabla \bar{p}+\nabla \cdot \underline{\underline{\underline{\sigma}}}-\nabla \cdot \underline{\underline{\sigma}}^{\mathrm{res}}+\bar{\rho} \boldsymbol{g},
\end{aligned}
$$

where $\bar{\rho}$ is the filtered density, $\widetilde{\boldsymbol{u}}$ is the filtered velocity vector, $\bar{p}$ is the filtered pressure, $\boldsymbol{g}$ is gravity vector, $\widetilde{\mathcal{D}}_{t}=\partial_{t}+\widetilde{\boldsymbol{u}} \cdot \nabla$ is the Favre-filtered substantial derivative, and $\underline{\underline{\sigma}}$ is the filtered viscous stress tensor with

$$
\underline{\underline{\sigma}}=2 \bar{\rho} \widetilde{\nu}\left[\underline{\underline{\widetilde{S}}}-\frac{1}{3}(\nabla \cdot \widetilde{\boldsymbol{u}}) \underline{\underline{I}}\right] \quad \text { and } \quad \widetilde{S}=\frac{1}{2}\left[\nabla \widetilde{\boldsymbol{u}}+(\nabla \widetilde{\boldsymbol{u}})^{T}\right]
$$


The residual stress tensor $\underline{\underline{\sigma}}^{\text {res }}=\bar{\rho} \widetilde{\boldsymbol{u u}}-\bar{\rho} \widetilde{\boldsymbol{u}} \widetilde{\boldsymbol{u}}$ is modeled by a dynamic Smagorinsky model. ${ }^{8,9}$ In deriving Eq. (4) a time-invariant filter is used, and commutation errors due to grid size variations are neglected. ${ }^{10}$ The density and molecular properties, appearing in Eqs. (4) and (5) are obtained through a state relation. However, instead of using the ideal gas law, which expresses $\rho$ in terms of pressure, temperature, and all species mass fractions, in the following a state equation is obtained from the flamelet/progress variable model.

\section{B. Flamelet/Progress Variable Model}

The flamelet/progress variable (FPV) model $^{11,12}$ is based on the flamelet equations, in which a turbulent diffusion flame is represented as an ensemble of laminar flame structures. ${ }^{13,14}$ For sufficiently large Damköhler number or sufficiently high activation energy, chemical reactions and heat transfer occur in a thin layer. If the characteristic length scale of this layer is small compared to that of the surrounding turbulence, turbulent structures are unable to penetrate into the reaction zone and cannot destroy the flame structure. Therefore, the effect of turbulence in this so-called flamelet regime results in a deformation and straining of the flame sheet.

The flamelet equations are obtained through a coordinate transformation of the transport equations for species mass fraction and temperature by introducing the mixture fraction $Z$ as independent variable. The unsteady flamelet equations can then be written as

$$
\partial_{t} \phi-\frac{\chi Z}{2} \partial_{Z}^{2} \phi=\dot{\omega}
$$

where $\dot{\boldsymbol{\omega}}$ corresponds to the source term of all species and temperature, which are collectively denoted by the vector $\phi$. The scalar dissipation rate of the mixture fraction, appearing in Eq. (6), is

$$
\chi_{Z}=2 \alpha|\nabla Z|^{2}
$$

and $\alpha$ is the mass diffusivity, which is assumed to be equal for all species. An analytical expression for $\chi_{Z}$ for a counter-flow diffusion flame was derived by Peters. ${ }^{15}$ This expression relates $\chi_{Z}$ to its value at stoichiometric condition and a function of $Z$ and $Z_{\mathrm{st}}$ :

$$
\chi_{Z}=\chi_{Z, \mathrm{st}} F(Z) \quad \text { with } \quad F(Z)=\exp \left\{2\left(\left[\operatorname{erfc}^{-1}\left(2 \mathrm{Z}_{\mathrm{st}}\right)\right]^{2}-\left[\operatorname{erfc}^{-1}(2 \mathrm{Z})\right]^{2}\right)\right\},
$$

and $\operatorname{erfc}^{-1}$ denotes the inverse of the complementary error function with $\operatorname{erfc}(\mathrm{Z})=1-\operatorname{erf}(\mathrm{Z})$.

The steady flamelet equations can be derived from Eq. (6) under the consideration that all species are formed on a sufficiently fast time scale, so that all species mass fractions and temperature are in quasi steady state, and the temporal derivative is negligible, resulting in

$$
-\frac{\chi_{Z}}{2} \partial_{Z}^{2} \phi=\dot{\omega}
$$

The solution of these equations can be represented by the so-called S-shaped curve, which is shown in Fig. 1(a). The upper and lower branches of this curve describe the stable burning and non-burning steady states, while the middle branch is unstable. The turning point between the upper and middle branches is denoted by $\chi_{Z, \mathrm{q}}$, corresponding to the critical scalar dissipation rate at which quenching occurs. Similarly, $\chi_{Z, \mathrm{i}}$ denotes the critical value below which an initially non-burning flamelet will ignite. Figure $1(\mathrm{~b})$ shows temperature profiles of three flamelets corresponding to a scalar dissipation rate of $\chi_{Z, \mathrm{st}}=100 \mathrm{~s}^{-1}$.

The steady flamelet structure can be computed from Eqs. (9), and all thermochemical quantities can then be parameterized in terms of mixture fraction and scalar dissipation rate, viz.,

$$
\psi=\mathcal{E}_{\psi}\left(Z, \chi_{Z, \mathrm{st}}\right),
$$

in which $\mathcal{E}_{\psi}$ corresponds to the steady-state flamelet relation, and $\boldsymbol{\psi}$ denotes all thermochemical quantities, including molecular properties, density, temperature, species mass fractions, and chemical source terms. The Favre-filtered form of this state relation can be used to provide information about density and molecular properties that are necessary to solve Eqs. (4). In addition to the governing equations describing conservation of mass and momentum, an additional transport equation for the mixture fraction must be solved, and the scalar dissipation rate can be obtained from an algebraic relation. Note, however, that this vertical projection 
(a) S-shaped Curve

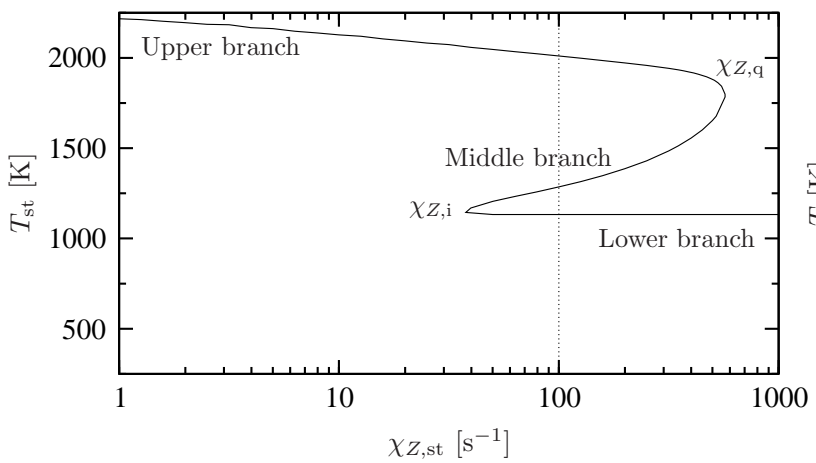

(b) Flamelet Solution

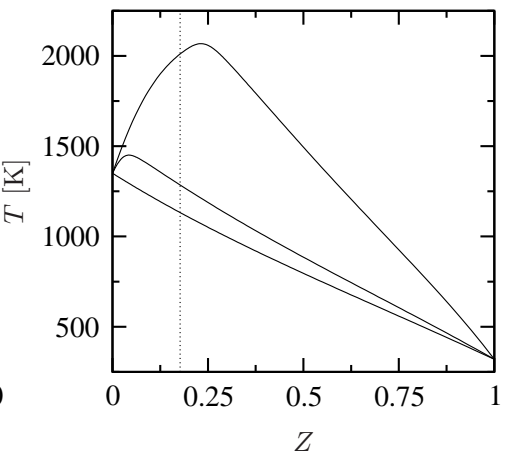

Figure 1. Solutions of the steady flamelet equations, Eqs. (9), for a methane/air flame, showing (a) temperature as function of the scalar dissipation rate at stoichiometric mixture fraction $Z_{\mathrm{st}}=0.177$; (b) Temperature profiles for three flamelets, computed for the same scalar dissipation rate $\chi_{Z \text {,st }}=100 \mathrm{~s}^{-1}$, and the vertical dashed line shows the location of stoichiometric mixture.

of all thermochemical quantities onto the $Z-\chi_{Z \text {,st }}$ plane is not unique and results in multiple solutions for $\chi_{Z, \mathrm{i}} \leq \chi_{Z \text {,st }} \leq \chi_{Z, \mathrm{q}}$. This can be seen in Fig. 1(b), showing temperature profiles for three flamelets evaluated for the same scalar dissipation rate of $\chi_{Z \text {,st }}=100 \mathrm{~s}^{-1}$.

To overcome the ambiguity of the steady flamelet model, a reaction progress parameter $\Lambda$ has been introduced in the FPV model. ${ }^{11,12}$ This mixture-fraction independent parameter, which is related to the reaction progress variable $C$, is defined so that each flamelet along the entire S-shaped curve can be uniquely identified. The reaction progress variable is defined from a linear combination of reaction products as $C=Y_{\mathrm{CO}}+Y_{\mathrm{CO}_{2}}+Y_{\mathrm{H}_{2} \mathrm{O}}+Y_{\mathrm{H}_{2}}$, and $\Lambda$, having a unique value for each flamelet, corresponds to $C$ evaluated at the stoichiometric condition:

$$
\Lambda=C \mid Z_{\mathrm{st}} .
$$

By replacing the scalar dissipation rate in Eq. (10) by $\Lambda$, the FPV state relation can be written as

$$
\psi=\mathcal{F}_{\psi}^{\mathrm{S}}(Z, \Lambda)
$$

and the superscript "S" refers to the steady flamelet equations. This relation is used instead of Eq. (10) to represent the complete thermochemical state space along the S-shaped curve.

Although this FPV model has been successfully applied to a wide range of combustion configurations, including flames with local extinction and reignition, ${ }^{16}$ due to the neglect of the transient term in the steady flamelet equations, this model is inadequate for the prediction of autoignition events. To overcome this shortcoming an unsteady flamelet/progress variable (UFPV) model is developed in the next section. This model extends the work by Pitsch \& Ihme ${ }^{3}$ by employing a statistically most-likely PDF to represent subgrid scale fluctuations of the reaction progress variable. The unsteady FPV model is presented in the next section, and the presumed PDF closure model is discussed in Sec. D.

\section{Unsteady Flamelet/Progress Variable Model for Autoignition}

Models employing the steady flamelet equations restrict the flamelet solution to that of the S-shaped curve. In fact, models in which all thermochemical quantities are parameterized in the form of Eq. (10) can only use a subset of the steady flamelet solution space, due to the non-uniqueness of the parameterization. Although the S-shaped curve represents the strong attractor and lower-dimensional manifold of the flamelet equations in the limit of sufficiently long residence time, for the consideration of transient and autoignition events, the complete state space must be considered, which is described by the unsteady flamelet equations.

An unsteady flamelet model, formulated in Lagrangian and Eulerian reference frame, was developed by Pitsch \& Steiner ${ }^{17}$ and Pitsch. ${ }^{18}$ In addition to the local scalar dissipation rate and mixture fraction, this model introduced a local flamelet time that is associated with the convection and diffusion of each flamelet. As such, the required information about the state-space trajectory and corresponding time for each flamelet limits the application of this model to canonical flows. 
The unsteady flamelet/progress variable (UFPV) model addresses this issue by expressing the flamelet time in terms of the reaction progress parameter and scalar dissipation rate. All thermochemical quantities are then parameterized in the form of

$$
\psi=\mathcal{F}_{\psi}^{\mathrm{U}}\left(Z, \Lambda, \chi_{Z, \mathrm{st}}\right)
$$

and the superscript "U" refers to the unsteady flamelet library. With this relation differential changes of a state-space variable $\boldsymbol{\psi}$ are then represented by

$$
d \boldsymbol{\psi}=\frac{\partial \boldsymbol{\psi}}{\partial Z} d Z+\frac{\partial \boldsymbol{\psi}}{\partial \Lambda} d \Lambda+\frac{\partial \boldsymbol{\psi}}{\partial \chi_{Z, \mathrm{st}}} d \chi_{Z, \mathrm{st}}
$$

Since this expression eliminates the time from the parameterization, it also assumes that the structure of a particular flamelet is independent from its history. In the context of the prediction of radiation and NO pollutant formation it was shown that this is indeed a valid assumption for species that evolve on sufficiently fast time scales. ${ }^{19}$ This, however, allows to populate the state space independently from a particularly flamelet trajectory. In the present application this is done as follows. First, the S-shaped curve is obtained from the solution of the steady flamelet equations. To obtain solutions 'inside' the S-shaped curve, starting with the initial conditions corresponding to the middle branch or a non-burning flamelet, the unsteady flamelet equations are solved for a specified scalar dissipation rate until the stable solution of the upper branch is reached. If the steady state solution is reached, the process is repeated with a different value for the scalar dissipation until the complete state space is populated. In this respect, the flamelet trajectory corresponds to a vertical line in the state space. Figure 2(a) illustrates the result of this procedure. The symbols correspond to the individual solutions of the unsteady flamelet equations that are obtained from the previously described method, and the solid line is the S-shaped curve from the steady flamelet equations.

(a) S-shaped Curve and Unsteady Flamelet Solutions

(b) Unsteady Flamelet Solution
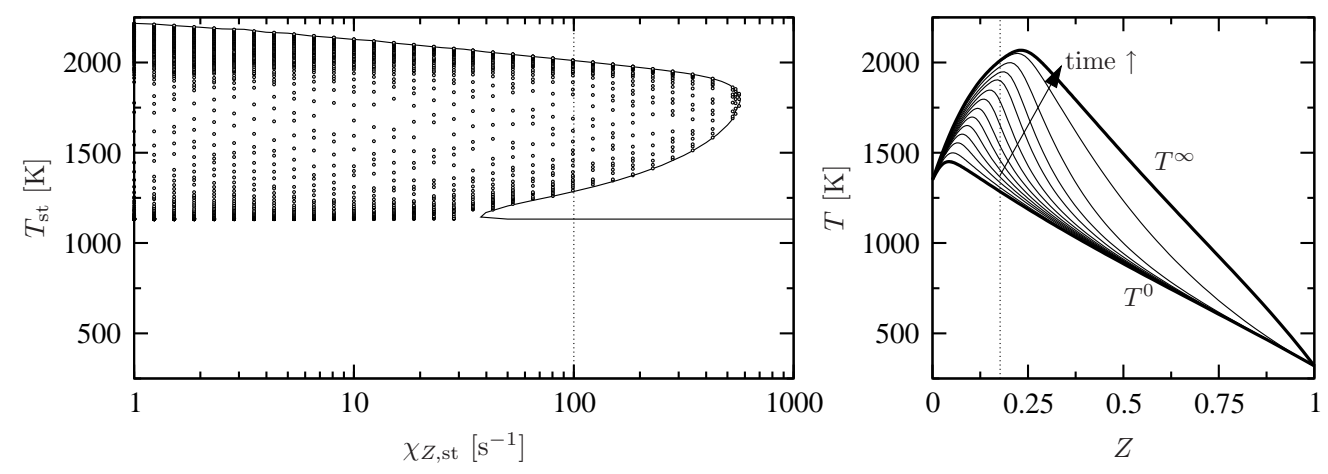

Figure 2. Solutions of the flamelet equations for a methane/air flame, showing (a) temperature as function of the scalar dissipation rate at stoichiometric mixture fraction $Z_{\mathrm{st}}=0.177$; the symbols correspond to solutions of the unsteady flamelet equations, and the solid line represents the S-shaped curve, obtained from the steady flamelet equations. (b) Temperature profiles, showing the evolution of a flamelet with increasing time, computed for a scalar dissipation rate of $\chi_{Z, \mathrm{st}}=100 \mathrm{~s}^{-1}$, and the vertical dashed line shows the location of the stoichiometric mixture fraction.

Figure 2(b) shows the temporal evolution of a flamelet for a constant scalar dissipation rate of $\chi_{Z \text {,st }}=100$ $\mathrm{s}^{-1}$. Beginning with a flamelet from the unstable middle branch, the flamelet ignites at a location at fuel-lean conditions, corresponding to the most-reactive mixture, ${ }^{20} Z_{\mathrm{MR}}$, and by diffusive effects in mixture fraction space, the flame structure evolves over about $2.5 \mathrm{~ms}$ until a steady-state condition is reached.

The ignition delay time $\tau_{\text {ig }}$ as function of $\chi_{Z \text {,st }}$ is shown in Fig. 3. Here, $\tau_{\text {ig }}$ is evaluated at stoichiometric conditions, and is defined as:

$$
\tau_{\mathrm{ig}}=\left\{t \mid T_{\mathrm{st}}(t)=\left(T_{\mathrm{st}}^{0}+T_{\mathrm{st}}^{\infty}\right) / 2\right\},
$$

where $T^{0}$ and $T^{\infty}$ denote the temperature profiles of the non-burning and steady burning flamelet solution, respectively. Beginning at the location corresponding to $\chi_{Z, \mathrm{i}}$, the ignition delay time decrease until it reaches a minimum of $\tau_{\mathrm{ig}}=7.1 \mathrm{~ms}$ at $\chi_{Z, \mathrm{st}}=5.75 \mathrm{~s}^{-1}$ and subsequently increases with decreasing scalar dissipation rate. 
Since the spatiotemporal structure of the flame is not fully resolved in LES and only described in a Favre-sense, a statistical description of the state relation (13) will be employed. For this, a presumed PDF closure is employed, which is described in the next section.

\section{Presumed PDF Closure Model}

For the LES prediction of turbulent reacting flows, the state relation (13) must be formulated for Favre-filtered quantities. In the following, Favre-filtered thermochemical quantities are computed from Eq. (13) by employing a presumed joint PDF for mixture fraction, reaction progress parameter, and stoichiometric scalar dissipation rate:

$$
\widetilde{\psi}=\iiint \mathcal{F}_{\psi}^{\mathrm{U}}\left(Z, \Lambda, \chi_{Z, \mathrm{st}}\right) \widetilde{P}\left(Z, \Lambda, \chi_{Z, \mathrm{st}}\right) d Z d \Lambda d \chi_{Z, \mathrm{st}},
$$

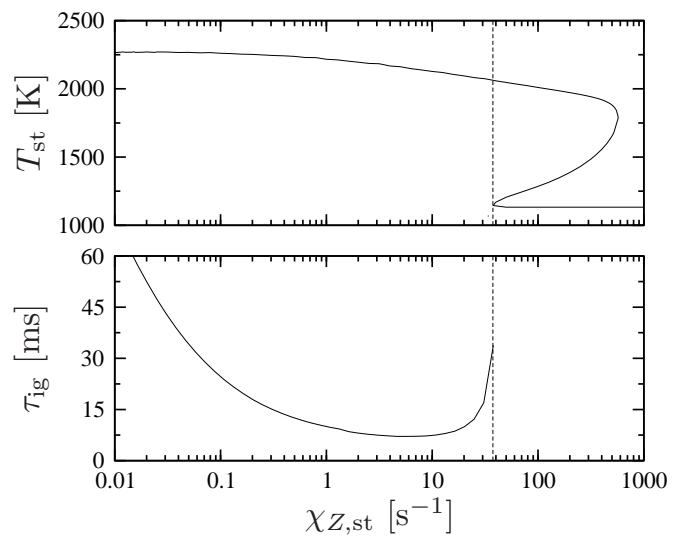

and $\widetilde{P}\left(Z, \Lambda, \chi_{Z, \text { st }}\right)$ denotes the density-weighted joint PDF with

$$
\widetilde{P}\left(Z, \Lambda, \chi_{Z, \mathrm{st}}\right)=\frac{\rho}{\bar{\rho}} P\left(Z, \Lambda, \chi_{Z, \mathrm{st}}\right)=\widetilde{P}\left(Z, \chi_{Z, \mathrm{st}}\right) P\left(\Lambda \mid Z, \chi_{Z, \mathrm{st}}\right) .
$$

Since $\Lambda$ is defined to be statistically independent from $Z$ and $\chi_{Z \text {,st }}$, the conditional $\operatorname{PDF} P\left(\Lambda \mid Z, \chi_{Z \text {,st }}\right)$ reduces to its marginal PDF. Furthermore, it is assumed that $Z$ and $\chi_{Z \text {,st }}$ are independent. With this, Eq. (17) can be written as

$$
\widetilde{P}\left(Z, \Lambda, \chi_{Z, \mathrm{st}}\right)=\widetilde{P}(Z) P\left(\chi_{Z, \mathrm{st}}\right) P(\Lambda) .
$$

A beta PDF is used to model the mixture fraction distribution, ${ }^{21,22}$ and the distribution of $\chi_{Z \text {,st }}$ is modeled by a delta function. ${ }^{3}$ A so-called statistically most-likely distribution ${ }^{23-26}$ (SMLD) is employed for $\Lambda$, so that $\widetilde{P}\left(Z, \Lambda, \chi_{Z, \text { st }}\right)$ can be written as

$$
\widetilde{P}\left(Z, \Lambda, \chi_{Z, \mathrm{st}}\right)=\beta\left(Z ; \widetilde{Z}, \widetilde{Z^{\prime \prime 2}}\right) \delta\left(\chi_{Z, \mathrm{st}}^{*}-\chi_{Z, \mathrm{st}}\right) P_{\mathrm{SML}, 2}(\Lambda)
$$

and

$$
P_{\mathrm{SML}, j}(\Lambda)=Q(\Lambda) \exp \left\{\sum_{i=0}^{j} a_{i} \Lambda^{i}\right\},
$$

where $j=2$ denotes the number of enforced moments, and $Q(\Lambda)$ is the so-called a priori $\mathrm{PDF},{ }^{27}$ accounting for bias in composition space. In the following, $Q$ is modeled as ${ }^{16}$

$$
Q(\Lambda)=\frac{1}{\sqrt{\widetilde{C^{\prime \prime 2}}}}\left[1+\left(\frac{\dot{\omega}_{C, \mathrm{st}} \gamma_{C} \widetilde{Z^{\prime \prime 2}}}{\chi_{Z, \mathrm{st}} \sqrt{C^{\prime \prime 2}}}\right)^{2}\right]^{1 / 2}
$$

and

$$
\gamma_{C}=\frac{\widetilde{\chi}_{Z}^{\mathrm{res}}}{\widetilde{Z^{\prime \prime 2}}} \frac{\widetilde{C^{\prime \prime 2}}}{\widetilde{\chi}_{C}^{\mathrm{res}}}
$$

is the frequency ratio between mixture fraction and progress variable. The weight coefficients $a_{i}$ in Eq. (20) are defined subject to the following constraints:

$$
\begin{aligned}
\iint \beta\left(Z ; \widetilde{Z}, \widetilde{Z^{\prime \prime 2}}\right) P_{\mathrm{SML}, 2}(\Lambda) d Z d \Lambda & =1 \\
\iint \mathcal{F}_{C}^{\mathrm{U}}\left(Z, \Lambda, \chi_{Z, \mathrm{st}}\right) \beta\left(Z ; \widetilde{Z}, \widetilde{Z^{\prime \prime 2}}\right) P_{\mathrm{SML}, 2}(\Lambda) d Z d \Lambda & =\widetilde{C} \\
\iint\left[\mathcal{F}_{C}^{\mathrm{U}}\left(Z, \Lambda, \chi_{Z, \mathrm{st}}\right)-\widetilde{C}\right]^{2} \beta\left(Z ; \widetilde{Z}, \widetilde{Z^{\prime \prime 2}}\right) P_{\mathrm{SML}, 2}(\Lambda) d Z d \Lambda & =\widetilde{C^{\prime \prime 2}}
\end{aligned}
$$


Note that in these equations, the $\chi_{Z \text {,st }}$-dependence is already integrated out. Equations (23) can be reformulated as a non-linear minimization problem, and can be solved iteratively ${ }^{28}$ for $\boldsymbol{a}=\boldsymbol{a}\left(\widetilde{Z}, \widetilde{Z^{\prime \prime 2}}, \widetilde{C}, \widetilde{C^{\prime \prime 2}}, \chi_{Z \text {,st }}\right)$, with $\boldsymbol{a}=\left(a_{0}, a_{1}, \ldots, a_{j}\right)^{T}$. After the coefficients $\boldsymbol{a}$ are determined, the joint $\operatorname{PDF} \widetilde{P}(Z, \Lambda, \chi Z$,st $)$ can be constructed and inserted into Eq. (16) to obtain the Favre-filtered form of the thermochemical state relation. In this context it is interesting to point out that the joint PDF is not directly dependent on the moment information about the reaction progress parameter, so that all Favre-filtered thermochemical quantities can be directly expressed in terms of $\widetilde{C}$ and $\widetilde{C^{\prime \prime 2}}$. This relation is here denoted by

$$
\widetilde{\psi}=\widetilde{\mathcal{G}}_{\psi}^{\mathrm{U}}\left(\widetilde{Z}, \widetilde{Z^{\prime \prime 2}}, \widetilde{C}, \widetilde{C^{\prime \prime 2}}, \chi_{Z, \mathrm{st}}\right) .
$$

Replacing the moments of the reaction progress parameter with moments of the progress variable introduces significant model simplifications, since the model complexity for the transport equations of $\widetilde{C}$ and $\widetilde{C^{\prime \prime 2}}$ is reduced.

In the following, Eq. (24) is used to provide information about all thermochemical quantities in the conservation equations for mass and momentum. In addition to the solution of Eqs. (4), four addition transport equations are required to close the system of equations in the UFPV model. The transport equations describing conservation of the first two moments of mixture fraction and progress variable can be written as

$$
\begin{aligned}
\bar{\rho} \widetilde{\mathcal{D}}_{t} \widetilde{Z} & =\nabla \cdot(\bar{\rho} \widetilde{\alpha} \nabla \widetilde{Z})+\nabla \cdot \tau_{\widetilde{Z}}^{\mathrm{res}}, \\
\bar{\rho} \widetilde{\mathcal{D}}_{t} \widetilde{Z^{\prime \prime 2}} & =\nabla \cdot\left(\bar{\rho} \widetilde{\alpha} \nabla \widetilde{Z^{\prime \prime 2}}\right)+\nabla \cdot \tau_{\widetilde{Z^{\prime \prime 2}}}^{\mathrm{res}}-2 \bar{\rho} \widetilde{\boldsymbol{u}^{\prime \prime} Z^{\prime \prime}} \cdot \nabla \widetilde{Z}-\bar{\rho} \widetilde{\chi}_{Z}^{\mathrm{res}}, \\
\bar{\rho} \widetilde{\mathcal{D}}_{t} \widetilde{C} & =\nabla \cdot(\bar{\rho} \widetilde{\alpha} \nabla \widetilde{C})+\nabla \cdot \tau_{\widetilde{C}}^{\mathrm{res}}+\bar{\rho} \widetilde{\dot{\omega}}_{C}, \\
\bar{\rho} \widetilde{\mathcal{D}}_{t} \widetilde{C^{\prime \prime 2}} & =\nabla \cdot\left(\bar{\rho} \widetilde{\alpha} \nabla \widetilde{C^{\prime \prime 2}}\right)+\nabla \cdot \tau_{C^{\prime \prime 2}}^{\mathrm{res}}-2 \bar{\rho} \widetilde{\boldsymbol{u}^{\prime \prime} C^{\prime \prime}} \cdot \nabla \widetilde{C}-\bar{\rho} \widetilde{\chi}_{C}^{\mathrm{res}}+2 \bar{\rho} \widetilde{C^{\prime \prime} \dot{\omega}_{C}^{\prime \prime}} \cdot
\end{aligned}
$$

The turbulent fluxes $\widetilde{\boldsymbol{u}^{\prime \prime} Z^{\prime \prime}}$ and $\widetilde{\boldsymbol{u}^{\prime \prime} C^{\prime \prime}}$ are modeled by a gradient transport assumption, and the term $\widetilde{C^{\prime \prime} \dot{\omega}_{C}^{\prime \prime}}$ is precomputed and stored in the flamelet library, Eq. (24). A model for the residual scalar dissipation rate of the mixture fraction is derived from spectral arguments, ${ }^{16}$ which is written as

$$
\widetilde{\chi}_{Z}^{\mathrm{res}}=\frac{C_{\chi_{Z}} C_{\epsilon}}{\mathrm{Sc}_{\mathrm{t}} C_{u}} \frac{\alpha_{\mathrm{t}}^{\widetilde{Z}}}{\Delta} \widetilde{Z^{\prime \prime 2}}
$$

where $S c_{t}=\nu_{t} / \alpha_{\mathrm{t}}^{\widetilde{Z}}$ is the turbulent Schmidt number for which a value of 0.4 was used. ${ }^{17}$ The expectation of $C_{\chi_{Z}}$ can be estimated using model energy spectra for turbulent kinetic energy and mixture fraction variance. Based on this analysis, a value of 2.0 was used for this coefficient. The ratio $C_{\epsilon} / C_{u}$ is dependent on the LES filter width $\Delta$, and rapidly approaches zero for increasing filter ratio $\Delta / \eta$, with $\eta$ denoting the Kolmogorov length scale. For the present application, a value of 0.5 was used for the ratio $C_{\epsilon} / C_{u}$. A closure model for $\tilde{\chi}_{C}^{\text {res }}$ is obtained from Eq. (22) by using Eq. (26) and using a specified value for $\gamma_{C}$, which is here set to 2.0.

\section{Experimental Configuration and Computational Setup}

The experiment used for validation of the autoignition model corresponds to the vitiated co-flow burner, which was experimentally studied by Cabra et al. ${ }^{4}$ The experimental setup consists of a central fuel pipe with a diameter of $D_{\text {ref }}=4.57 \mathrm{~mm}$, through which a methane/air mixture at a temperature of $320 \mathrm{~K}$ is supplied. The jet exit velocity is $U_{\text {ref }}=100 \mathrm{~m} / \mathrm{s}$. The Reynolds number based on the fuel nozzle diameter, exit velocity, and kinematic viscosity of the fuel mixture is 24,200 , and the value of the stoichiometric mixture fraction is $Z_{\mathrm{st}}=0.177$. The co-flow consists of reaction products from a premixed hydrogen/air combustion. It is reported that the product mixture, consisting of oxygen, nitrogen, and water, is uniform across the co-flow stream, and the temperature is $1,350 \mathrm{~K}$. The co-flow has a diameter of $210 \mathrm{~mm}$ and is surrounded by an exit collar to prevent entrainment of ambient air into the flame. The experimental parameters are summarized in Tab. 1.

The Favre-filtered governing equations are solved in cylindrical coordinates. ${ }^{11}$ The geometry is nondimensionalized by the jet nozzle diameter $D_{\text {ref }}$ and the computational domain is $90 D_{\text {ref }} \times 30 D_{\text {ref }} \times 2 \pi$ in axial, radial, and circumferential directions, respectively. The axial direction is discretized with 256 grid 
Table 1. Reference parameters for the lifted jet flame simulation.

\begin{tabular}{|cccc|}
\hline Parameter & Units & Jet & Co-flow \\
\hline \hline$d$ & $\mathrm{~m}$ & $4.57 \times 10^{-3}\left(=D_{\text {ref }}\right)$ & 0.210 \\
$U$ & $\mathrm{~m} / \mathrm{s}$ & $100\left(=U_{\text {ref }}\right)$ & 5.4 \\
$T$ & $\mathrm{~K}$ & 320 & 1,350 \\
$X_{\mathrm{O}_{2}}$ & - & 0.15 & 0.12 \\
$X_{\mathrm{N}_{2}}$ & - & 0.52 & 0.73 \\
$X_{\mathrm{H}_{2} \mathrm{O}}$ & - & - & 0.15 \\
$X_{\mathrm{CH}}$ & - & 0.33 & - \\
$Z_{\text {st }}$ & - & 0.177 & \\
\hline
\end{tabular}

points following a linear growth rate, and 150 grid points are used in radial direction. The circumferential direction is equally spaced and uses 64 points, resulting in a total number of approximately 2.5 million grid points. The minimum and maximum filter widths are $\Delta_{\min }=4 \times 10^{-2} D_{\text {ref }}$ (at the centerline near the nozzle exit) and $\Delta_{\max }=1.27 D_{\text {ref }}$ (outermost computational cell at the exit plane). For reference, the grid stretching diagram is shown in Fig. 4.

The turbulent inflow velocity profile was generated from a periodic pipe flow simulation. The unsteady flamelet calculations have been performed using the FlameMASTER code,${ }^{29}$ and the chemistry is described by the GRI 2.11 mechanism. ${ }^{30}$ From the unsteady flamelets, the UFPV flamelet library is generated. To increase the table resolution, $\widetilde{Z^{\prime \prime 2}}$ was replaced by the mixedness, $\widetilde{S}=\widetilde{Z^{\prime \prime 2}} /\left(\widetilde{Z}-\widetilde{Z}^{2}\right)$, and the grid stretching in the directions of $\widetilde{Z}, \widetilde{S}$, and $\widetilde{C^{\prime \prime 2}}$ followed a geometric series. For the discretization of the chemistry table, 75 points are used for the $\widetilde{Z}$ and $\widetilde{C}$ directions, 20 points are used in the directions of $\widetilde{S}$ and $\widetilde{C^{\prime \prime 2}}$, and 15 points were used for $\chi_{Z, \mathrm{st}}$.
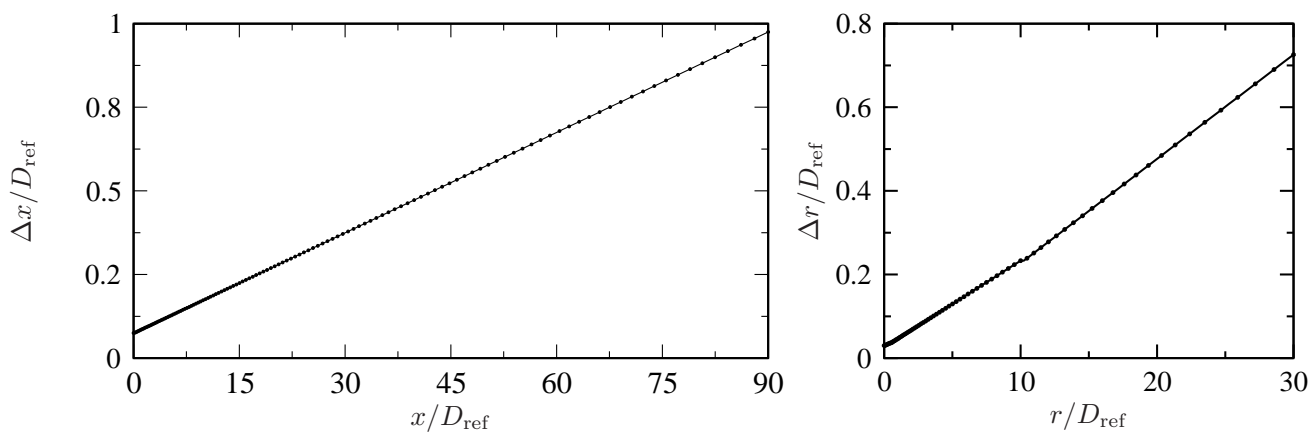

Figure 4. LES grid stretching diagram: (left) axial direction; (right) radial direction. Only every second grid point is shown by the symbols.

\section{Results}

\section{A. Instantaneous Flow Field}

The UFPV model was applied to LES of the Cabra et al. flame, ${ }^{4}$ and in the following the simulation results are compared with experimental data. An additional simulation was conducted using the steady FPV model, and differences between both models are discussed. Statistical results, denoted by angular brackets, are obtained from azimuthal and temporal averaging of the instantaneous flow field quantities, and Favre-averaged quantities are computed as $\{\widetilde{\psi}\}=\langle\bar{\rho} \widetilde{\psi}\rangle /\langle\widetilde{\psi}\rangle$.

The instantaneous and averaged temperature fields obtained from both models are shown in Fig. 5 . The solid lines in these figures correspond to the isocontour of the stoichiometric mixture fraction. From the instantaneous temperature field obtained from the simulation with the UFPV model in Fig. 5(a), it can be seen that up to approximately $25 D_{\text {ref }}$ downstream of the jet exit fuel and oxidizer mix without significant heat release. Following this inert mixing zone, a transition region between $30 \leq x / D_{\text {ref }} \leq 50$ is apparent, 


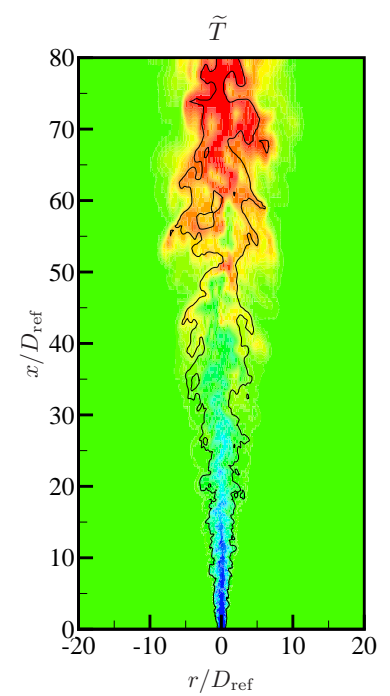

(a) UFPV Model
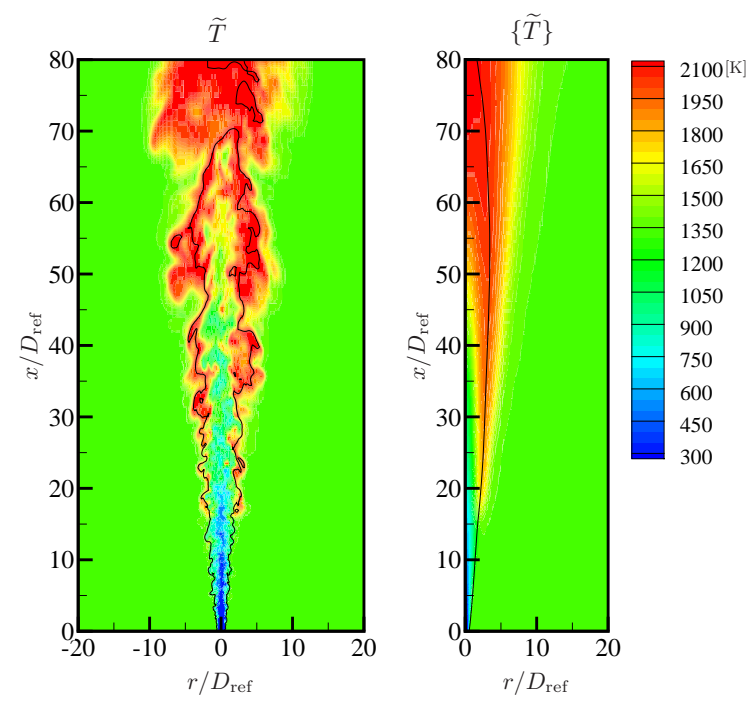

(b) FPV Model

Figure 5. Instantaneous and averaged temperature fields obtained from (a) UFPV model and (b) FPV model. The solid line shows the location of stoichiometric mixture fraction.

in which the temperature increases; however, some intermittent pockets with low temperature are evident. Beyond a distance of 50 nozzle diameters above the jet exit the flame is continuously burning, and some entrainment of fluid from the co-flow into the flame core can be observed from the instantaneous flow field results. The lift-off height was computed from the averaged flow field results using the same metric as described by Gordon et al. ${ }^{5}$ The predicted lift-off height of $35 D_{\text {ref }}$ is in reasonable agreement with the experimentally reported value of $30 D_{\text {ref }}$. Note, however, that the experimentally determined value was based on the ethylene and acetylene concentrations, which could partially be responsible for the discrepancy.

The prediction obtained from the steady FPV model shows a significantly different flame behavior. After a considerable reduction of the inert mixing region, the flame rapidly ignites at approximately $15 D_{\text {ref }}$, and a transition region, as observed for the UFPV simulation, is not existing. The steady FPV model significantly underpredicts the lift-off height with a value of $16 D_{\text {ref }}$.

(a) UFPV Model

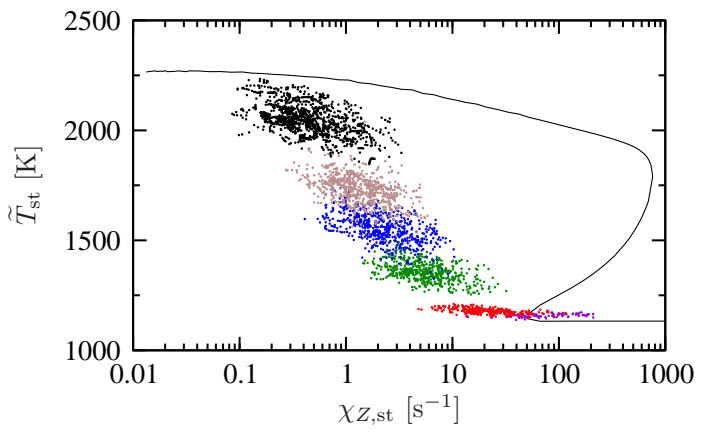

(b) FPV Model

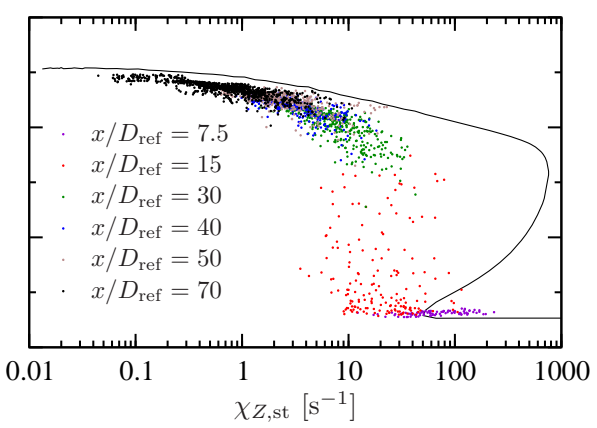

Figure 6. Scatter plot of Favre-filtered temperature as function of stoichiometric scalar dissipation rate obtained from (a) UFPV model and (b) steady FPV model. For reference the S-shaped curve is shown by the solid line.

Single point data at six different axial locations in the jet flame are sampled from both simulations. These data are presented in the form of scatter plots in Fig. 6. For reference, the steady flamelet solution of the S-shaped curve is also shown. The differences in the flame structure predictions, discussed in the context of Fig. 5, are emphasized by these mixture-fraction conditioned scatter plots. While individual flamelets 
in the UFPV simulation exhibit a transient evolution that fills a large portion of the flamelet space, the flamelets from the FPV simulation are primarily confined to the upper stable branch of the S-shaped curve. As previously pointed out, the FPV model predicts a faster flame ignition, which is due to the fact that all thermodynamic flame state are projected onto the S-shaped curve. It is also interesting to point out the strong fluctuations in the stoichiometric scalar dissipation rate for all measurement location, that typically spans more than an order in magnitude.

\section{B. Statistical Flow Field Results}

Favre-averaged results for mixture fraction and temperature along the jet centerline are shown in Fig. 7 . Apart from the slight overprediction in the transition region for $25 \leq x / D_{\text {ref }} \leq 60$ the prediction of the mean mixture fraction from the UFPV model is in excellent agreement with the experimental data. Note that the FPV model considerably overpredicts $\{\widetilde{Z}\}$ in the transition region, and also the mixture fraction fluctuations from the FPV model exhibit deviations from the experimental measurements.

The mean temperature from the UFPV model is in overall good agreement with experimental data. The model predicts an initially faster temperature rise in the ignition region, which is further delayed in the downstream direction. A detailed analysis of the results suggests that this delay of the ignition process can be attributed to larger values of the progress variable variance, and the predictions would benefit from improvements in the model for $\tilde{\chi}_{C}^{\text {res }}$.
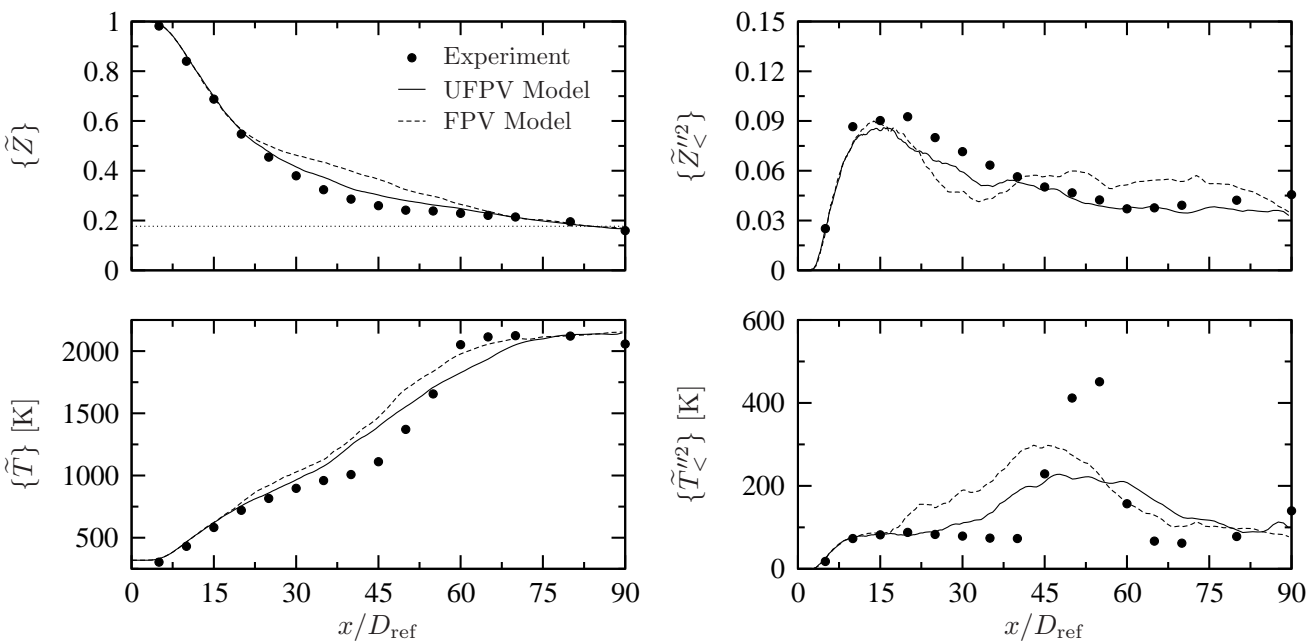

Figure 7. Comparison of measured (symbols) and calculated (lines) mean and rms statistics of mixture fraction and temperature along the centerline for the Cabra-flame.

The mean temperature as function of mean mixture fraction is illustrated in Fig. 8, showing the differences between both models. Although the UFPV model overpredicts the ignition process in the transition region, the model shows significant improvements over the steady FPV model.

Radial profiles for the predictions of mixture fraction, temperature, and species mass fractions of $\mathrm{CO}_{2}$, $\mathrm{CO}$, and $\mathrm{H}_{2} \mathrm{O}$ are compared with experimental data in Fig. 9. Favre-averaged mixture fraction profiles are shown in the first row, and the results from the UFPV model are in better agreement with experimental data compared to the FPV results. As already discussed in the context of Fig. 5, the steady FPV model predicts an early flame ignition process, which is not observed in the experimental measurements. Compared to these results, the UFPV model accurately captures the temperature evolution in the transition region; however, the location of the peak temperature is slightly shifted towards the centerline. This discrepancy can be attributed to the overprediction of the mean mixture fraction profiles (see first row) and also to the shortened autoignition, which moves the temperature peak towards fuel-richer composition (see Fig. 2(b)).

The major product mass fractions of $\mathrm{CO}_{2}$ and $\mathrm{H}_{2} \mathrm{O}$ are in similarly good agreement than the temperature profiles, and the spatial evolution of the species is well predicted by the UFPV model. The radial profiles for the intermediate carbon monoxide species show considerable differences between experiment and prediction. The reason for these discrepancies are currently under investigation. 


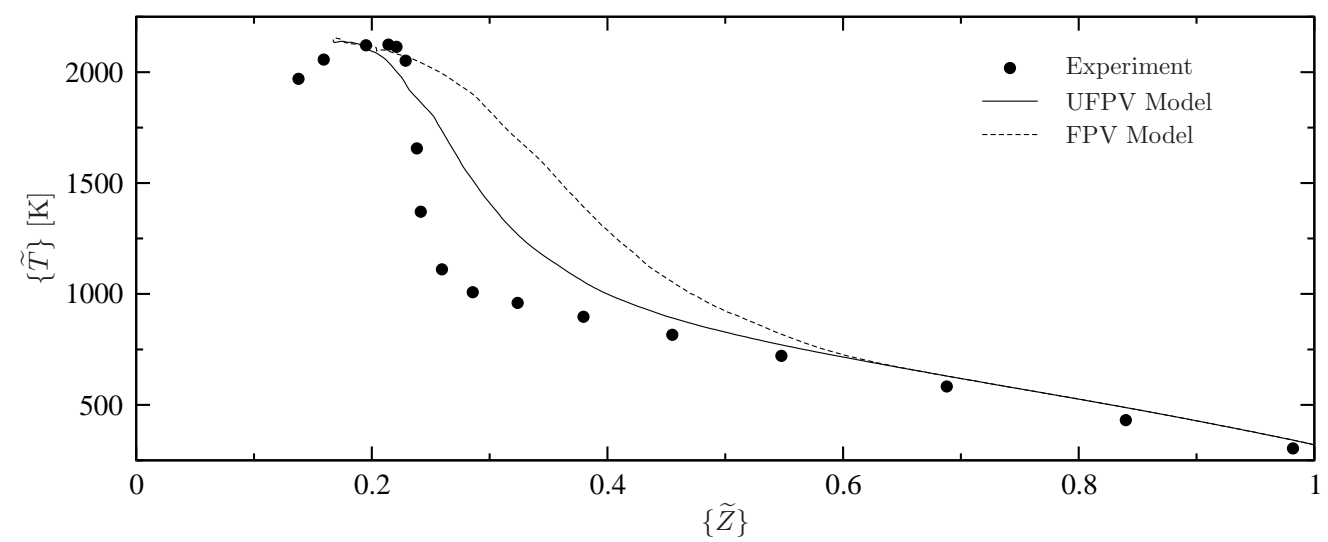

Figure 8. Comparison of the predicted mean temperature as a function of $\{\tilde{Z}\}$ along the centerline with experimental data.

\section{Conditional Flow Field Results}

Mixture fraction conditioned results for temperature and species mass fractions of $\mathrm{CO}_{2}, \mathrm{CO}, \mathrm{H}_{2} \mathrm{O}$, and $\mathrm{OH}$ are illustrated in Fig. 10. Statistical results for the conditional temperature, presented in the first row, show that the UFPV model predicts a slightly faster ignition process up to $x / D_{\text {ref }}=40$, which is then followed by a delay for $x / D_{\text {ref }}>50$. Compared to these results, the steady FPV model predicts a fully burning flame state already at $x / D_{\text {ref }}=30$ with slowly increasing peak temperature for increasing downstream direction due to the decrease in the scalar dissipation rate. Conditional results for temperature and mass fractions of $\mathrm{CO}_{2}$ and $\mathrm{H}_{2} \mathrm{O}$, evaluated at the last measurement station, also suggest that the flame reaches steady-state conditions. Conditional data for the hydroxyl radical, illustrated in the last row, show that the UFPV model qualitatively predicts the peak location and formation process of $\mathrm{OH}$ up to $x / D_{\text {ref }}=50$, but underpredicts the $\mathrm{OH}$ consumption at the last measurement location.

Overall, the conditional results for temperature and species mass fractions predicted with the UFPV model are in reasonable agreement with experimental data, and the model adequately captures the transient flame ignition process.

\section{Conclusion}

An unsteady flamelet/progress variable model has been developed for predicting the ignition process in a lifted flame. The model is an extension to the steady flamelet/progress variable approach, and employs the unsteady flamelet model to describe the evolution of all thermochemical quantities during the flame ignition process. In the UFPV model, all thermochemical quantities are then parameterized by mixture fraction, reaction progress parameter, and stoichiometric scalar dissipation rate. The particular advantage of this model over previously developed unsteady flamelet formulations is that in the UFPV model the flamelet time is replaced by physical quantities, which leads to significant simplifications in the computation and parameterization of the thermodynamic state space.

A presumed PDF closure model is employed to evaluate Favre-averaged thermochemical quantities. For this a beta-distribution is used for the mixture fraction, a statistically most-likely distribution is employed for the reaction progress parameter, and the distribution of the stoichiometric scalar dissipation rate is modeled by a Dirac delta function.

The UFPV model was applied to LES of a lifted flame in a vitiated co-flow, and simulation results are compared with experimental data and results obtained from the steady FPV model. Compared to the steady FPV model, it is demonstrated that the unsteady formulation leads to significantly improved predictions for flame structure, lift-off height, and spatiotemporal evolution of the flow field. Although the UFPV model predicts a slightly faster ignition behavior, mixture fraction and temperature fields are in good agreement with experimental data.

Mixture-fraction conditioned data for temperature, major species, and $\mathrm{OH}$ mass fraction are analyzed. In addition, scatter plots for the stoichiometric temperature are compared between both models, showing 

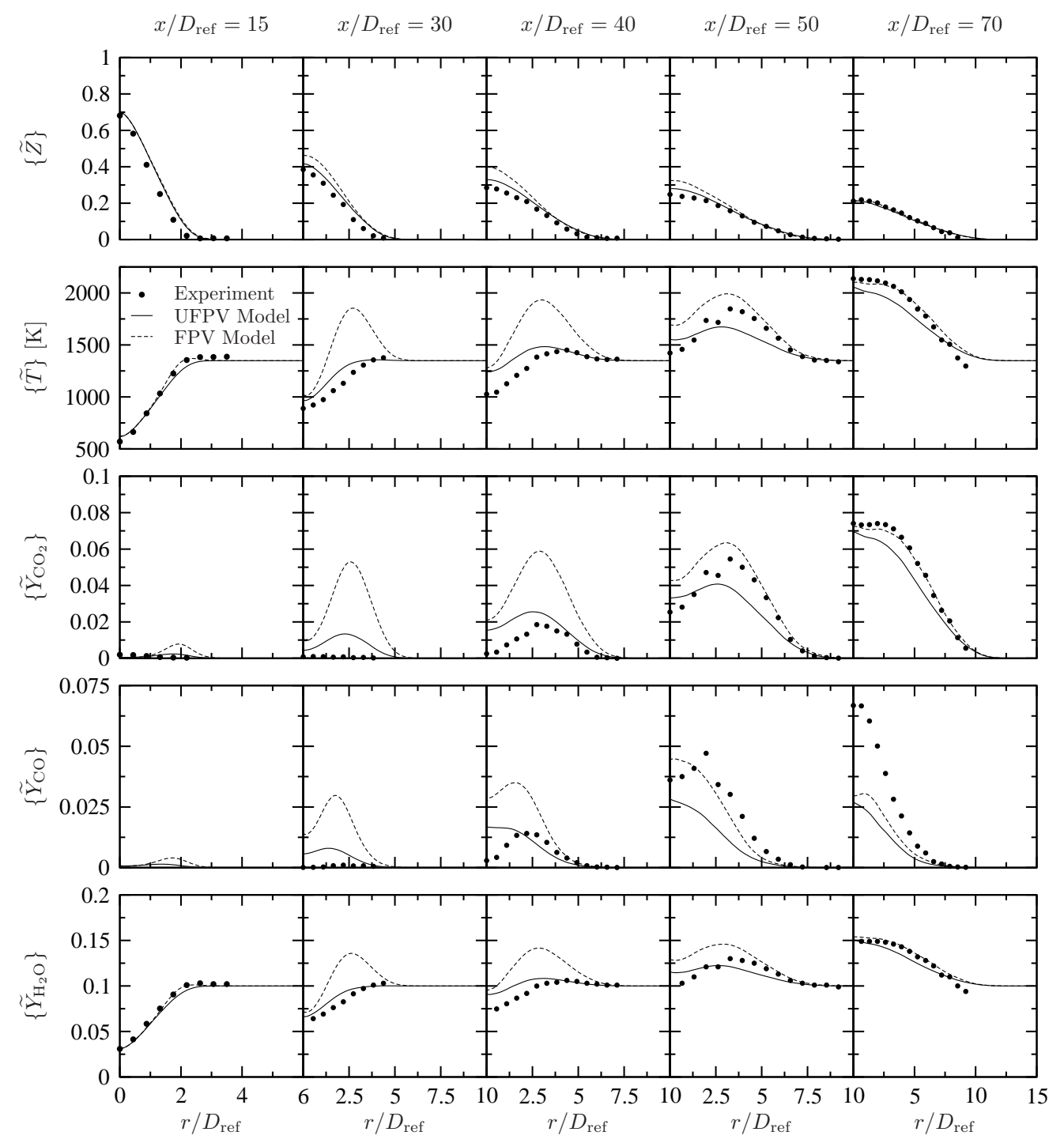

Figure 9. Comparison of radial profiles between simulations and experiments, for mixture fraction, temperature, and species mass fractions of $\mathrm{CO}_{2}, \mathrm{CO}$, and $\mathrm{H}_{2} \mathrm{O}$.

that the UFPV model occupies a large portion of the unsteady flamelet space, while the scatter data for the FPV model are primarily confined to the upper stable branch of the S-shaped curve, which explains the short ignition time and reduction in the lift-off height.

\section{References}

${ }^{1}$ Lefebvre, A. H., Gas Turbine Combustion, Taylor \& Francis, New York, 1999.

${ }^{2}$ Pitsch, H., "Large-eddy simulation of turbulent combustion," Ann. Rev. Fluid Mech., Vol. 38, 2006, pp. 453-482.

${ }^{3}$ Pitsch, H. and Ihme, M., "An unsteady/flamelet progress variable method for LES of nonpremixed turbulent combustion," 43rd AIAA Aerospace Sciences Meeting and Exhibit, paper AIAA-2005-557, Jan. 2005, Reno, NV, USA.

${ }^{4}$ Cabra, R., Chen, J.-Y., Dibble, R. W., Karpetis, A. N., and Barlow, R. S., "Lifted methane-air jet flames in a vitiated coflow," Combust. Flame, Vol. 143, 2005, pp. 491-506.

${ }^{5}$ Gordon, R. L., Masri, A. R., Pope, S. B., and Goldin, G. M., "Transport budget in turbulent lifted flames of methane autoigniting in a vitiated co-flow," Combust. Flame, Vol. 151, 2007, pp. 495-511.

${ }^{6}$ Gkagkas, K. and Lindstedt, R. P., "Transported PDF modeling with detailed chemistry of pre- and auto-ignition in $\mathrm{CH}_{4}$ /air mixtures," Proc. Combust. Inst., Vol. 31, 2007, pp. 1559-1566.

${ }^{7}$ Domingo, P., Vervisch, L., and Veynante, D., "Large-eddy simulation of a lifted methane jet flame in a vitiated coflow," Combust. Flame, Vol. 152, 2008, pp. 415-432. 

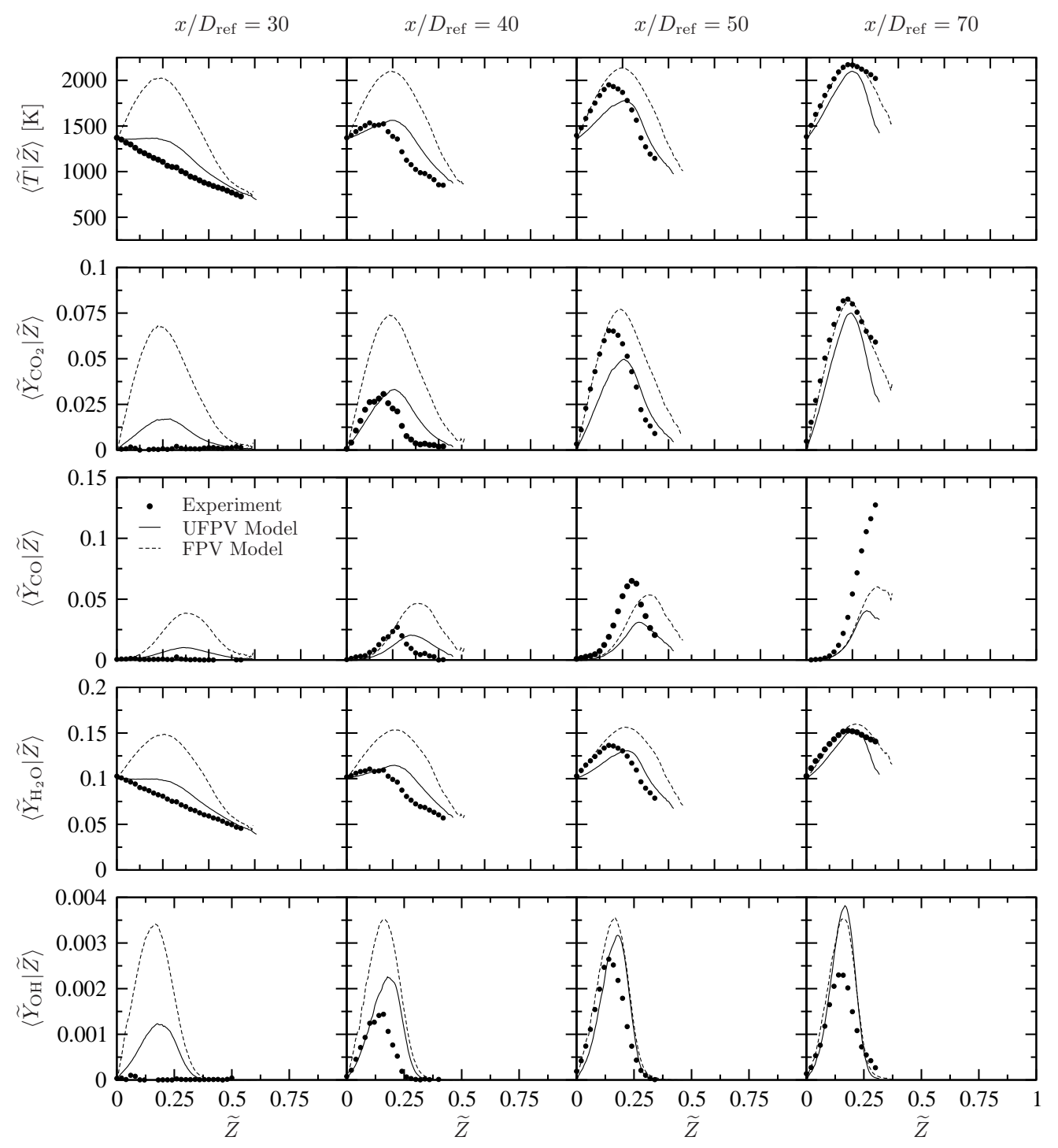

Figure 10. Comparison of measured (symbols) and calculated (lines) conditional mean temperature and mean mass fractions of $\mathrm{CO}_{2}, \mathrm{CO}, \mathrm{H}_{2} \mathrm{O}$, and $\mathrm{OH}$ at four axial locations in the flame.

${ }^{8}$ Germano, M., Piomelli, U., Moin, P., and Cabot, W. H., "A dynamic subgrid-scale eddy viscosity model," Phys. Fluids A, Vol. 3, No. 7, 1991, pp. 1760-1765.

${ }^{9}$ Lilly, D. K., "A proposed modification of the Germano subgrid-scale closure method," Phys. Fluids A, Vol. 4, No. 3, 1992, pp. 633-635.

${ }^{10}$ Ghosal, S., "An analysis of numerical errors in large eddy simulations of turbulence," J. Comp. Phys., Vol. 125, No. 1, 1996, pp. 187-206.

${ }^{11}$ Pierce, C. D. and Moin, P., "Progress-variable approach for large-eddy simulation of non-premixed turbulent combustion," J. Fluid Mech., Vol. 504, 2004, pp. 73-97.

${ }^{12}$ Ihme, M., Cha, C. M., and Pitsch, H., "Prediction of local extinction and re-ignition effects in non-premixed turbulent combustion using a flamelet/progress variable approach," Proc. Combust. Inst., Vol. 30, 2005, pp. 793-800.

${ }^{13}$ Peters, N., "Laminar diffusion flamelet models in non-premixed turbulent combustion," Prog. Energy Combust. Sci., Vol. 10, No. 3, 1984, pp. 319-339.

${ }^{14}$ Peters, N., "Local quenching due to flame stretch and non-premixed turbulent combustion," Combust. Sci. Tech., Vol. 30, 1983, pp. 1-17.

${ }^{15}$ Peters, N., Turbulent Combustion, Cambridge University Press, Cambridge, 2000.

${ }^{16}$ Ihme, M. and Pitsch, H., "Prediction of extinction and reignition in non-premixed turbulent flames using a flamelet/progress variable model. 2. A posteriori study with application to Sandia flames D and E," Combust. Flame, Vol. 155, 2008, pp. 90-107. 
${ }^{17}$ Pitsch, H. and Steiner, H., "Large-eddy simulation of a turbulent piloted methane/air diffusion flame (Sandia flame D)," Phys. Fluids, Vol. 12, No. 10, 2000, pp. 2541-2554.

${ }^{18}$ Pitsch, H., "Improved pollutant predictions in large-eddy simulations of turbulent non-premixed combustion by considering scalar dissipation rate fluctuations," Proc. Combust. Inst., Vol. 29, 2002, pp. 1971-1978.

${ }^{19}$ Ihme, M. and Pitsch, H., "Modeling of Radiation and NO Formation in Turbulent Non-premixed Flames Using a Flamelet/Progress Variable Formulation," Phys. Fluids, Vol. 20, 2008, pp. 055110.

${ }^{20}$ Liñán, A. and Crespo, A., "An asymptotic analysis of unsteady diffusion flames for large activation energies," Combust. Sci. Tech., Vol. 14, No. 1-3, 1976, pp. 95-117.

${ }^{21}$ Wall, C., Boersma, B. J., and Moin, P., "An evaluation of the assumed beta probability density function subgrid-scale model for large eddy simulation of nonpremixed, turbulent combustion with heat release," Phys. Fluids, Vol. 12, No. 10, 2000, pp. $2522-2529$.

22 Jiménez, J., Linãń, A., Rogers, M. M., and Higuera, F. J., "A priori testing of subgrid models for chemically reacting non-premixed turbulent shear flows," J. Fluid Mech., Vol. 349, 1997, pp. 149-171.

${ }^{23}$ Jaynes, E. T., "Information theory and statistical mechanics," Phys. Rev., Vol. 106, No. 4, 1957, pp. 620-630.

${ }^{24}$ Shannon, C. H., "A mathematical theory of communication," Bell System Tech. J., Vol. 27, No. 3, 1948, pp. 379-423.

${ }^{25}$ Good, I. J., "Maximum-entropy for hypothesis formulation, especially for multidimensional contingency-tables," Ann. Math. Statist., Vol. 34, No. 3, 1963, pp. 911-934.

${ }^{26}$ Pope, S. B., "A rational method of determining probability distributions in turbulent reacting flows," J. Non-Equilib. Thermodyn., Vol. 4, 1979, pp. 309-320.

${ }^{27}$ Fang, C.-S., Rajasekera, J. R., and Tsao, H.-S. J., Entropy Optimization and Mathematical Programming, Kluwer Academic Publisher, Boston, 1997.

${ }^{28}$ Ihme, M. and Pitsch, H., "Prediction of extinction and reignition in non-premixed turbulent flames using a flamelet/progress variable model. 1. A priori study and presumed PDF closure," Combust. Flame, Vol. 155, 2008, pp. 7089.

${ }^{29}$ Pitsch, H., "FlameMaster v3.1: A C++ computer program for 0D combustion and 1D laminar flame calculations," 1998, available from http://www.stanford.edu/ ${ }^{\text {hpitsch/. }}$

${ }^{30}$ Bowman, C. T., Hanson, R. K., Davidson, D. F., Gardiner, W. C., Lissianski, V., Smith, G. P., Golden, D. M., Frenklach, M., and Goldenberg, M., "GRI-Mech 2.11," 1997, available from http://www.me.berkeley.edu/gri-mech/. 